\title{
The benefits of flash glucose monitoring in the UK
}

\author{
MASA JOSIPOVIC, ${ }^{1}$ MARK EVANS ${ }^{2}$
}

A century after the life-transforming discovery and purification of insulin, many people living with type 1 diabetes (T1D) are not reaching glycaemic goals. ${ }^{1,2}$ Therapeutic approaches to help people with T1D achieve glucose targets and (equally importantly) reduce the burden of living with diabetes include structured education, new insulins and technology for delivering insulin, measuring glucose, decision support and closed loop technology to automate insulin delivery. The Freestyle Libre was first launched in Europe in 2014 with uptake in UK use having increased since it was made available on NHS prescription in 2017. There have been changes from the original device with the introduction of an algorithm to improve accuracy and the launch of the second generation Libre2 device allowing the optional use of alarms. Current UK T1D penetrance is around 50\% in England (with a marked increase since April 2019 facilitated by NHS England as part of the NHS Long Term Plan), with higher rates in Scotland, Wales and Northern Ireland. A recent Health Technology Wales guidance has recommended broader use for all people with insulin-treated diabetes, not just T1D. ${ }^{3}$

Worldwide use is also increasing markedly. This global growth in the use of the Libre has occurred despite the lack of a supporting body of evidence from randomised controlled trials (RCTs) showing lowering of $\mathrm{HbA}_{1 \mathrm{c}}$, the traditional outcome metric for diabetes trials. ${ }^{4}$ For readers' interest, an RCT (FLASH-UK) has been examining this in the UK, with participants with T1D randomised to Libre2 versus control finger prick testing. ${ }^{5}$ At the time of writing, FLASHUK had just completed follow-up and the results are eagerly anticipated. Despite the current absence of RCT data, there are, however, many real-world observations showing improved clinical outcomes with the Libre. ${ }^{6}$ In the UK, data show reductions in $\mathrm{HbA}_{1 c}$ and a striking reduction in severe hypoglycaemia and diabetic ketoacidosis with use of the Libre in Scotland. ${ }^{7}$ The Association of British Clinical Diabetologists ( $A B C D$ ) has been running nationwide audits of medications introduced into real-world use in the UK since

Gates Trust Cambridge Scholar/ PhD Student, Wellcome Trust/ MRC Institute of Metabolic Science, University of Cambridge, Cambridge UK 2 University Professor of Diabetic Medicine and Honorary Consultant Physician, Wellcome Trust/ MRC Institute of Metabolic Science \& Department of Medicine, University of Cambridge, Cambridge UK

Address for correspondence: Professor Mark Evans IMS MRL Box 289, Addenbrooke's Hospital, Hills Road, Cambridge CB2 OQQ, UK

E-mail:mle24@medschl.cam.ac.uk

Br J Diabetes 2021;21:284-285

https://doi.org/10.15277/bjd.2021.323
2004. An $A B C D$ audit of Libre outcomes has been running since 2017, reporting reduced $\mathrm{HbA}_{1 c}$, improved hypoglycaemia awareness and reductions in hospital attendances for dysglycaemia. ${ }^{8}$

This edition of the journal contains an examination of data from the $A B C D$ Libre audit, asking whether prior structured education affects the outcomes with flash glucose monitoring. In particular, clinical outcomes were compared between those who had undergone Dose Adjustment for Normal Eating (DAFNE) structured education, other structured education or neither. Structured education to support self-management of T1D includes a variety of programmes across the UK and elsewhere with variable approaches/ quality assurance, evidence and governance/structure. ${ }^{9}$ DAFNE is currently delivered in 99 centres and based on principles of therapeutic education with a written curriculum, multidisciplinary team working with defined accreditation, quality assurance and RCT and real-world evidence for efficacy. 10,11 This includes (but is not limited to) equipping participants with the ability to appraise and utilise glucose information judiciously.

A priori, it would have been possible to hypothesise that those undergoing structured education/DAFNE might be better placed to interpret and benefit from more comprehensive glucose data provided by the Libre. An alternative hypothesis would be that those who had undergone structured education had already partbenefited from the ability to interpret glucose information and would have less incremental gain from the Libre. Of note, there is a large repository of free online training (including the Diabetes Technology Network-UK resources cited in the paper) targeted specifically at how to use and interpret Libre data which would have been available to all regardless of previous structured education and, indeed, many services would have encouraged or even mandated evidence that people had undergone this more targeted training.

The study included 14,880 patients, stratified into three groups based on prior structured education status: 4,215 DAFNE graduates, 3,964 other structured education graduates and 6,701 patients who had not received structured education. The main outcomes were the impact of previous education on glycaemic control assessed by $\mathrm{HbA}_{1 c}$ levels, and hypoglycaemia awareness measured by the standardised GOLD score. At follow-up, all three groups showed improvements from Libre initiation, with reduced $\mathrm{HbA}_{1 \mathrm{c}}$ (by $8.10 \mathrm{mmol} / \mathrm{mol}, 6.61 \mathrm{mmol} /$ $\mathrm{mol}$ and $6.22 \mathrm{mmol} / \mathrm{mol}$, respectively) and GOLD score (by 0.33 , 0.30 and 0.34 , respectively). There was no statistical difference between groups in terms of the magnitude of these changes ( $p=0.83$ for $\mathrm{HbA}_{1 c}, \mathrm{p}=0.42$ for GOLD). Interestingly, on linear regression modelling, the authors show that a higher baseline 
$\mathrm{HbA}_{1 c}$, frequency of flash monitoring and other structured education (but not DAFNE) were predictors of $\mathrm{HbA}_{1 \mathrm{c}}$ reduction.

What does this mean for clinicians? Firstly, the authors and indeed all who have contributed to the ongoing $A B C D$ audit are to be congratulated. This is an impressive repository of data. As with any real-world data, there are advantages and drawbacks compared with RCT data. Perhaps unsurprisingly, there were significant and clinically meaningful differences between groups. Those who had undergone DAFNE training were older with a longer duration of diabetes and insulin pump use. Readers should be cautious in assigning too much weight to group comparisons.

Taken together, we suggest that there are two key 'take-home' messages from this paper. First, it does not show that flash glucose monitoring means that structured education is now a vestigial offering. DAFNE in particular continues to empower people with T1D to self-manage their diabetes and, based on the supporting evidence, was the only structured education programme specifically cited in NICE NG17 T1D guidelines. ${ }^{12}$ Second, although of critical importance, access to structured education should not be regarded as an impediment to receiving and benefiting from blood glucose monitoring technology. All groups benefited from FLASH with reductions in $\mathrm{HbA}_{1 \mathrm{c}}$ and improvements in hypoglycaemia awareness scores. Not all are able to access and/or willing to commit time to undergoing structured education despite changes to make programmes like DAFNE more accessible including remote access. This paper clearly shows that this group still benefit from access to the Libre. We suggest that structured education and technology are complementary tools in the growing clinical armamentarium to support people living with T1D. The case for widespread use of flash glucose monitoring for all with insulin-treated diabetes across the whole of the UK continues to grow!

Conflict of interest MLE is a member of the national DAFNE executive and a trialist in the FLASH-UK study. He has received honoraria for advisory boards and speakers' fees from Abbott Diabetes Care (manufacturer of Freestyle Libre). MJ reports no conflicts of interest.

Funding None.

\section{References}

1. McKnight JA, Wild SH, Lamb MJE, et al. Glycaemic control of type 1 diabetes in clinical practice early in the 21st century: an international comparison. Diabet Med 2015;32(8):1036-50. https://doi.org/10.1111/ dme.12676
2. Shah VN, Grimsmann JM, Foster NC, et al. Undertreatment of cardiovascular risk factors in the type 1 diabetes exchange clinic network (United States) and the prospective diabetes follow-up (Germany/Austria) registries. Diabetes Obes Metab 2020;22(9):1577-85. https://doi.org/10.1111/ dom.14069

3. Health Technology Wales (HTW) Guidance 004-2 (July 2021). https://www. healthtechnology.wales/wp-content/uploads/2021/ 09/GUI004-FlashGM-FINAL.pdf

4. Secher AL, Pedersen-Bjergaard U, Svendsen OL, et al. Flash glucose monitoring and automated bolus calculation in type 1 diabetes treated with multiple daily insulin injections: a 26 week randomised, controlled, multicentre trial. Diabetologia 2021;64(12):2713-24. https://doi.org/10.1007/ s00125-021-05555-8

5. Wilmot EG, Evans M, Barnard-Kelly K, et al. Flash glucose monitoring with the FreeStyle Libre 2 compared with self-monitoring of blood glucose in suboptimally controlled type 1 diabetes: the FLASH-UK randomised controlled trial protocol. BMJ Open 2021;11(7):e050713. https://doi.org/ 10.1136/bmjopen-2021-050713

6. Evans M, Welsh Z, Ells S, Seibold A. The impact of flash glucose monitoring on glycaemic control as measured by $\mathrm{HbA1c}$ : a meta-analysis of clinical trials and real-world observational studies. Diabetes Ther Res Treat Educ Diabetes Relat Disord 2020;11(1):83-95. https://doi.org/10.1007/s13300019-00720-0

7. Jeyam A, Gibb FW, McKnight JA, et al. Flash monitor initiation is associated with improvements in $\mathrm{HbA} 1 \mathrm{c}$ levels and DKA rates among people with type 1 diabetes in Scotland: a retrospective nationwide observational study. Diabetologia 2021 Oct 7. https://doi.org/10.1007/s00125-021-05578-1. [Online ahead of print].

8. Deshmukh H, Wilmot EG, Choudhary P, et al. Impaired awareness of hypoglycemia and severe hypoglycemia in drivers with diabetes: insights from the Association of British Clinical Diabetologists Nationwide Audit. Diabetes Care 2021;44(11):e190-e191. https://doi.org/10.2337/dc211181

9. Heller SR, Gianfrancesco C, Taylor C, Elliott J. What are the characteristics of the best type 1 diabetes patient education programmes (from diagnosis to long-term care), do they improve outcomes and what is required to make them more effective? Diabet Med 2020;37(4):545-54. https://doi.org/10.1111/dme.14268

10. Hopkins D, Lawrence I, Mansell P, et al. Improved biomedical and psychological outcomes 1 year after structured education in flexible insulin therapy for people with type 1 diabetes: The UK DAFNE experience. Diabetes Care 2012;35(8):1638-42. https://doi.org/10.2337/dc11-1579

11. DAFNE Study Group. Training in flexible, intensive insulin management to enable dietary freedom in people with type 1 diabetes: dose adjustment for normal eating (DAFNE) randomised controlled trial. BMJ 2002; 325(7367):746. https://doi.org/10.1136/bmj.325.7367.746

12. National Institute for Health and Care Excellence. Type 1 diabetes in adults: diagnosis and management. NICE Guideline 17. 2015. https://www.nice.org.uk/guidance/ng17 